\title{
UN ESTUDIO AL IMPACTO DE LAS INICIATIVAS DE MEJORA DE LAS CADENAS DE SUMINISTRO Y DE FABRICACIÓN EN EMPRESAS QUE APLICAN MTO Y MTS
}

\author{
Ariel L. Lituve, Anna Merdie \\ The University of Adelaide, Australia \\ researchboard@adelaide.edu.au
}

(Tipo de artículo: INVESTIGACIÓN. Recibido el 30/07/2011. Aprobado el 15/11/2011)

\begin{abstract}
RESUMEN
Las empresas manufactureras tienen como objetivo mejorar los procesos internos y externos para incrementar la ventaja competitiva. Estos procesos incluyen prácticas de manufactura esbelta, de racionalización de proveedores y de integración logística. En este trabajo, se analizan estas prácticas y su impacto en el desempeño del negocio y, en particular, se exploran las diferencias entre las empresas que aplican make-to-order -MTO- y make-to-stock -MTS-, con los datos recolectados de 216 empresas manufactureras de Australia. En los resultados se encontró una clara diferencia del enfoque de mejora entre las empresas MTO y las MTS. Las MTO muestran un impacto significativo de la integración logística en el desempeño empresarial, pero no de las prácticas de manufactura esbelta y de racionalización de proveedores. La situación se revierte completamente para las empresas MTS que, aunque logran efectos significativos en las prácticas internas de manufactura esbelta y en la racionalización de proveedores, no lo logran en la integración logística. Además, que la distinción entre empresas MTO y MTS es importante cuando se analizan las iniciativas para mejorar las cadenas de fabricación y de suministro.
\end{abstract}

Palabras clave

Punto de desacoplamiento, prácticas de manufactura esbelta, integración logística, desempeño de negocio, racionalización de proveedores.

\section{A STUDY ON THE IMPACT OF IMPROVEMENT INITIATIVES OF SUPPLY AND MANUFACTURING CHAINS IN COMPANIES APPLYING MTO AND MTS}

\begin{abstract}
Manufacturing companies have as objective the improvement of internal and external processes to increase the competitive advantage. These processes include lean manufacturing practices, rationalization of providers and logistics integration. In this work, these practices and their impact on business performance are analyzed and particularly, the differences between companies applying make-to-order -MTO- and make-to-stock -MTS- are explored, with the data collected from 216 manufacturing companies in Australia. In the results it was found a clear difference of the improvement approach between MTO and MTS companies. The MTO's companies show a significant impact of logistics integration in business performance, but not of lean manufacturing practices and providers' rationalization. The situation is completely different for MTS companies, although they achieve significant impact on the internal practices of lean manufacturing and in the rationalization of the providers, they do not achieve it in logistics integration. Furthermore, the distinction between MTO and MTS companies is important when analyzing initiatives to improve the production and supply chains.
\end{abstract}

\section{Keywords}

Decoupling point, lean practices, logistics integration, business performance, rationalization of suppliers.

\section{ÉTUDE SUR L'IMPACT DES INITIATIVES D'ÁMELIORATION DES CHAÎNES D'APPROVISIONEMENT ET DE FABRICATION DANS ENTREPRISES QU'APPLIQUENT MTO ET MTS}

\begin{abstract}
RÉSUMÉ
L'objectif des entreprises de manufacture est l'amélioration des processus internes et externes pour accroître l'avantage compétitif. Ces processus compris pratiques de manufacture Lean, de rationalisation de fournisseurs et d'intégration logistique. Dans ce travail, on analyse ces pratiques et son impact sur la performance de l'entreprise, et en particulier, on explore les différences entre les entreprises qu'appliquent make-to-order (fabrication à la commande) -MTO- et make-to-stock (production sur stocks )-MTS-, avec les données recueillies de 216 entreprises de manufacture Australiennes. Dans les résultats on a trouvé une différence évidente de l'approche d'amélioration entre les entreprises MTO et MTS. Les entreprises type MTO montrent un impact important de l'intégration logistique sur la performance des entreprises, mais pas des pratiques de manufacture Lean et de rationalisation de fournisseurs. La situation est complètement contraire pour les entreprises type MTS, qui en dépit de réussir des effets importants sur les pratiques internes de manufacture Lean et sur la rationalisation des fournisseurs, ne réussissent pas ça sur l'intégration logistique. De plus, la différenciation entre entreprises MTO et MTS est important quand on analyse les initiatives pour améliorer les chaînes de fabrication et approvisionnement.
\end{abstract}

Mots-clés

Point de découplage, pratiques de manufacture Lean, intégration logistique, performance de l'entreprise, rationalisation des fournisseurs. 


\section{INTRODUCCIÓN}

Las empresas manufactureras cada vez más entienden que las ganancias en una ventaja competitiva no se limitan a mejoras en sus operaciones internas sino que también deben tener en cuenta las cadenas de suministro externas. Existe una amplia gama de potenciales iniciativas de mejora para dar forma a los procesos internos y externos, como las prácticas de manufactura externa, integración logística y racionalización de proveedores. Las prácticas de manufactura esbelta son un ejemplo de iniciativa interna de mejora de procesos, mientras que las otros dos son ejemplos de iniciativas orientadas externamente. Este tipo de prácticas y programas generalmente se consideran que son beneficiosas para cualquier tipo de empresa manufacturera, mientras que algunos investigadores sugieren que otras iniciativas de mejora son más aplicables en ciertos entornos de fabricación. Por lo tanto, existe cierto desacuerdo. Además, no se encontraron investigaciones empíricas previas a gran escala que contrastarán los diferentes tipos de empresas y que explorarán las potenciales diferencias en este contexto.

La producción de manufactura esbelta generalmente se considera como un programa fundamental para cualquier empresa que desee mejorar sus operaciones de fabricación, por ejemplo, eliminando residuos y creando un flujo de producción sin problemas [1]. Sin embargo, existe un flujo de búsqueda "esbelto y ágil" que contrasta manufactura esbelta con ágil, lo que sugiere que la producción de manufactura esbelta es aplicable para operaciones MTS y para incrementar el punto de desacoplamiento en el flujo de materiales de la cadena de suministros, mientras que la agilidad se recomienda para las operaciones MTO y para disminuir el punto de desacoplamiento [2].

La integración logística se considera una parte vital para cualquier empresa en un contexto de cadena de suministro. Con el fin de crear una cadena de suministro fuerte, se requiere integrar la logística entre los socios sucesivos y muchos investigadores lo consideran importante para las operaciones de la cadena de suministro en general [3]. Sin embargo, otros sugieren que esto es particularmente cierto para las empresas MTO, que confían en el soporte de sus proveedores para proporcionar creación de variantes de componentes y para entregar productos personalizados a tiempo [4]; mientras que las empresas MTS compran componentes estándar y prefieren relaciones de partes independientes con los proveedores, por lo que no requieren la integración logística con ellos [5]. Por lo tanto, la producción de manufactura esbelta puede ser más aplicable para operaciones MTS y la integración logística más aplicable para operaciones MTO. Consecuentemente, debe ser importante y fructífero explorar si existen tales diferencias.

La racionalización de proveedores es una iniciativa de mejora que, de acuerdo con la literatura [6], parece ser de aplicación general. Una base de suministro limitada puede ser beneficiosa para ambas operaciones MTO y MTS, siendo capaz de concentrarse en unos pocos proveedores que ofrecen artículos y componentes de calidad y entregas confiables. Por consiguiente, en este trabajo se incluye la racionalización de proveedores para tener un conjunto completo de alternativas relacionadas con operaciones MTO y MTS: una que puede ser más aplicable para operaciones MTO -integración logística-, una que puede ser más aplicables para operaciones MTS prácticas de manufactura esbelta- y una que parece aplicable tanto para operaciones MTO como MTS racionalización de proveedores.

No se encontró ninguna investigación previa mediante encuesta que contrastará empresas MTO y MTS y que explorará las diferencias potenciales. En este trabajo se asume una visión de teoría de contingencia, en la que se investiga si el impacto de las iniciativas de mejora para la cadena de fabricación y de suministro, sobre el desempeño del negocio, es fortuito cuando la planta opera sobre una base MTO o sobre una MTS. El objetivo es contribuir a la investigación sobre puntos de desacoplamiento proporcionando los resultados de la encuesta acerca de las diferencias entre ambas empresas.

En primer lugar, se presentan los fundamentos teóricos y la literatura relacionada y se discuten las hipótesis. A continuación, se presenta la metodología de investigación y los resultados obtenidos. Luego, se discuten las implicaciones para los directores e investigadores $y$, finalmente, se relacionan las conclusiones.

\section{FUNDAMENTOS TEÓRICOS E HIPÓTESIS}

\subsection{Enfoque de la contingencia}

La teoría de la contingencia [7, 8], sostiene que ninguna teoría o método se puede aplicar en todas las instancias [9, 10]. En otras palabras, que no existe una forma ideal de organizarse para alcanzar resultados efectivos y que el énfasis en resultados pueden variar de una organización a otra [11]. Esto sugiere que las organizaciones deben hacer coincidir sus estructuras y procesos con el entorno, a fin de maximizar el desempeño [7, 12].

Aunque la teoría de la contingencia se ha aplicado a otras áreas de la gestión de operaciones -sobre todo, en la estrategia de fabricación-, pequeñas aplicaciones han tenido lugar en el estudio de las prácticas de la gestión de operaciones [9]. En particular, Sousa \& Voss [9] identifican sólo un ejemplo relacionado con MTO o MTS y Hendry [13], quien discute la política para satisfacer la demanda del cliente -make-to-order vs otras políticas- y hace recomendaciones basadas en casos relativos a cómo adaptar las prácticas a un contexto particular -maketo-order/job.

Estudios de caso previos basados en investigaciones [13-16] han encontrado que las operaciones MTO son diferentes de las operaciones MTS y que los investigadores de modelos matemáticos [17-20] han modelado situaciones MTO y MTS de manera diferente. Por lo tanto, es importante explorar las 
potenciales diferencias en las prácticas de la gestión de operaciones entre MTO y MTS en una encuesta.

En este artículo se explora si diferentes iniciativas de mejora impactan de forma diferente el desempeño del negocio sobre una orientación al mercado de producción en términos de MTO vs MTS. Al hacerlo, se tiene en cuenta a Sousa \& Voss [9] para identificar y consolidar las variables de contingencia relevantes.

\subsection{Desempeño del negocio}

El uso del desempeño del negocio como una medida de rendimiento es común para capturar el comportamiento a largo plazo de la empresa [21-25]. Por lo general, la cuota de mercado, el rendimiento de las inversiones, los activos y las ventas, o medidas similares como el cambio en estas medidas se utilizan para capturar el rendimiento empresarial. Por lo tanto, estas medidas tienen que ver tanto con el mercado como con el desempeño financiero. La razón por la que las empresas MTO y MTS utilizan el desempeño del negocio en lugar del rendimiento operativo puede localizarse en diferentes prioridades competitivas y en resultados de rendimiento operativo, mientras que el desempeño del negocio no está asociado con un determinado tipo de punto de desacoplamiento -tal como MTO o MTS. Por ejemplo, las empresas MTS típicamente compiten en precios y costos eficientes, mientras que las empresas MTO compiten en personalización y flexibilidad. En consecuencia, ambas empresas pueden utilizar diferentes prácticas o caminos para llegar a altos niveles de desempeño, pero ambos tipos de empresas deben ser capaces de lograr el buen desempeño empresarial. Por lo tanto, el desempeño del negocio se puede utilizar para medir el impacto de las iniciativas en las empresas manufactureras, con independencia del punto de desacoplamiento.

\subsection{Prácticas internas de manufactura esbelta}

El origen del término "manufactura esbelta" se remonta al International Motor Vehicle Program [26] y se presentó, por primera vez, como un sinónimo para las prácticas aplicadas en Toyota [27]. Sin embargo, los conceptos y las técnicas bajo la etiqueta de manufactura esbelta eran básicamente las mismas que "justo a tiempo" en la década anterior [28]. Womack \& Jones [1] proporcionaron cinco principios para la manufactura esbelta: 1) el valor, que lo define el cliente final, 2) la cadena de valor, que es el conjunto de todas las actividades específicas que se requieren para producir un producto específico a través de la cadena de valor interna, 3) el flujo, para darle valor a la creación, 4) utilizar un programa apropiado y 5) mejorar continuamente. Siguiendo estos principios, las prácticas internas de manufactura esbelta incluyen: reducir la configuración, un apropiado sistema de producción, reducir el tamaño de las piezas y racionalizar la distribución a través de, por ejemplo, fabricación celular o conceptos de fábrica focalizados [29-31]. Más específicamente, se trata de prácticas relacionadas internamente, en lugar de clientes 0 proveedores relacionados [30,31]. Varios estudios han encontrado evidencia de que la mejora del desempeño del negocio, en general, se asocia con el uso de métodos de justo a tiempo y de manufactura esbelta; también se observan incrementos en lo financiero [3235] y en el funcionamiento del mercado [35, 36]. Por eso, la primera hipótesis que se desarrolla en esta investigación tiene que ver con el impacto de las prácticas internas de manufactura esbelta en el desempeño empresarial.

Hipótesis 1: Las prácticas internas de manufactura esbelta tienen una relación positiva con el desempeño del negocio de la organización.

\subsection{Integración logística externa}

La creciente competencia ha llevado a las empresas no sólo a mejorar sus operaciones internas -por ejemplo, las prácticas de manufactura esbelta-, sino también a focalizarse en la integración de sus proveedores a los procesos de la cadena de valor global. También se reconoce ampliamente la contribución de los proveedores en la entrega de valor a los clientes, desde la que se construyen capacidades competitivas -calidad, distribución, flexibilidad y costo. La esencia de la integración logística es un flujo bien coordinado de materiales desde los proveedores [37]. La mejora de la integración logística entre los socios de la cadena de suministro se utiliza para obtener una serie de beneficios operacionales así como para mejorar en el servicio al cliente y en las ventas [38]. Detoni \& Nassimbeni [39] encontraron que la mejora en el desempeño de las organizaciones presenta un mayor nivel de interacción logística; Frohlich \& Westbrook [37] encontraron que un arco más amplio de interacciones logísticas estaba asociado fuertemente con el mejoramiento del desempeño y Flynn et al. [10] encontraron una relación significativa con el desempeño del negocio. En una revisión de estudios empíricos sobre la integración de la cadena de suministro [3] se encontró que la mayoría de los estudios reportan una positiva relación entre la integración y el desempeño y que aproximadamente la mitad de los encuestados utilizan el desempeño del negocio.

Hipótesis 2: La integración logística externa tiene una relación positiva con el desempeño del negocio de la organización.

\subsection{Racionalización de proveedores}

La racionalización de proveedores es un componente importante en la asociación estratégica con los proveedores y se refiere a la práctica de limitar la base de suministro a unos pocos proveedores estratégicos que pueden proveer alta calidad y fiabilidad [6, 40]. Eliminar el exceso le permite a la empresa conformar alianzas eficaces con aquellos que están dispuestos a producir componentes de alta calidad y a bajo costo [41]. Yu et al. [42] hicieron un estudio comparativo entre compras a un único proveedor o a varios en un entorno con riesgos de interrupción en la cadena de suministro y encontraron que tanto uno como dos proveedores pueden ser eficaces, dependiendo de la magnitud de la probabilidad de la interrupción. Chen y Paulraj [6] presentan un resumen de aspectos y beneficios relacionados con la reducción de la base de proveedores, incluyendo mejor penetración en el mercado. En un estudio de caso relativo a la selección y racionalización de proveedores usando análisis 
envolvente de datos, Narasimhan et al. [43] encontraron que la racionalización de proveedores tuvo un efecto positivo sobre el crecimiento del negocio en la compañía objeto del estudio.

Hipótesis 3: La racionalización de proveedores tiene una relación positiva con el desempeño del negocio de la organización.

\subsection{Make-to-order y make-to-stock}

Prácticas internas de manufactura esbelta para MTO y MTS. El concepto de "esbeltez", introducido por Naylor et al. [2], sugiere que la manufactura esbelta se debe enfatizar en las operaciones MTS, mientras que la agilidad es útil para operaciones MTO [2, 44-46]. Riezebos et al. [47] reconocen que las empresas MTS enfrentan más problemas que las empresas MTO o ETO (engineer-to-order), lo que ha tenido gran impacto en el desarrollo de mecanismos de control de producción para los sistemas de producción de manufactura esbelta. Ellos argumentan que Kanban está limitado a empresas MTS, mientras que CONWIP y POLCA son preferibles en entornos MTO. En general, los sistemas de producción repetitiva parecen ser más avanzados para utilizar las prácticas JIT/manufactura esbelta que los sistemas de producción no repetitiva, obteniendo una mayor utilización de estas prácticas en comparación con los sistemas no repetitivos [48]. Del mismo modo, Cusumano [49] encontró que las compañías de manufactura esbelta han experimentado problemas cuando se enfrentan con situaciones como "mucha variedad de productos" o "'muchas opciones de oferta para los clientes'", lo que frecuentemente origina "órdenes muy pequeñas y muy raras". Esta variedad requiere ajustes frecuentes e intercambio de equipos Kanban, así como muchas entregas de pequeños lotes de componentes. Como solución a este problema, Cusumano [49] propone que las empresas de manufactura esbelta deben reducir la variedad y utilizar partes más estandarizadas. La gran variedad de productos es una característica típica de las empresas MTO, mientras que la estandarización de partes es una característica típica de las empresas MTS. Por lo tanto, las prácticas internas de manufactura esbelta parecen más aplicables para entornos MTS.

Hipótesis 4: Las prácticas internas de manufactura esbelta tienen una relación positiva fuerte con el desempeño del negocio de la organización en empresas MTS y débil en empresas MTO.

Integración logística externa para MTO y MTS. Una mayor integración de la cadena de suministro en organizaciones MTS, mediante el intercambio de información y la coordinación del flujo físico, ofrece importantes oportunidades para mejorar el rendimiento económico [4]. Mientras que el intercambio de información reduce costos, el principal beneficio económico proviene de coordinar la toma de decisiones [4]. Quesada et al. [5] encontraron que las empresas con altos niveles de integración externa fueron las que lideraron en distribución, servicio al cliente, calidad y flexibilidad, en comparación con las empresas con baja integración, predominantemente focalizadas en los precios. Consecuentemente, las empresas que buscan distribución, servicio al cliente, calidad y flexibilidad para los pedidos deben centrarse en la manera de integrar externamente a sus clientes y a sus proveedores [5]. Frohlich \& Westbrook [37] encontraron que una mayor integración de la cadena de suministro externa genera mayores niveles de mejora en los tiempos de entrega y en las entregas a tiempo. Zailani \& Rajagopal [50] presentaron los resultados de un estudio en empresas de EE.UU. y Japón, donde la flexibilidad mejora luego de llevar a cabo la integración de la cadena de suministro externa. Así, la integración logística externa parece más aplicable para entornos MTO que compiten en flexibilidad y tiempos de entrega.

Hipótesis 5: En empresas MTO, la integración logística externa tiene una relación positiva más fuerte con el desempeño del negocio de la organización que para empresas MTS.

Racionalización de proveedores para MTO y MTS. Las empresas MTO necesitan proveedores confiables y artículos de alta calidad -con tiempos de entrega cortos y fiables y alta flexibilidad de diseño- para soportar la integración logística entre proveedores y compradores. Las empresas MTS necesitan proveedores confiables y artículos de alta calidad -a precios asequibles- para soportar los procesos internos de manufactura esbelta que han desarrollado. No se encontró ninguna investigación que sustentará decididamente que la reducción de la base de proveedores debe ser más relevante para un tipo de punto de desacoplamiento. Por lo tanto, el impacto de la racionalización de proveedores en el desempeño del negocio se supone que es positivo tanto para empresas MTO como MTS.

Hipótesis 6: La racionalización de proveedores tiene una relación positiva con el desempeño del negocio tanto en empresas MTO como en empresas MTS.

\section{MODELO DE LA INVESTIGACIÓN}

El marco de esta investigación se muestra en la Figura 1. Las prácticas internas de manufactura esbelta, la integración logística externa y la racionalización de proveedores se modelan como antecedentes potenciales del desempeño del negocio. El modelo se puso a prueba para toda la muestra y para dos submuestras, diferenciando entre empresas MTO y MTS.

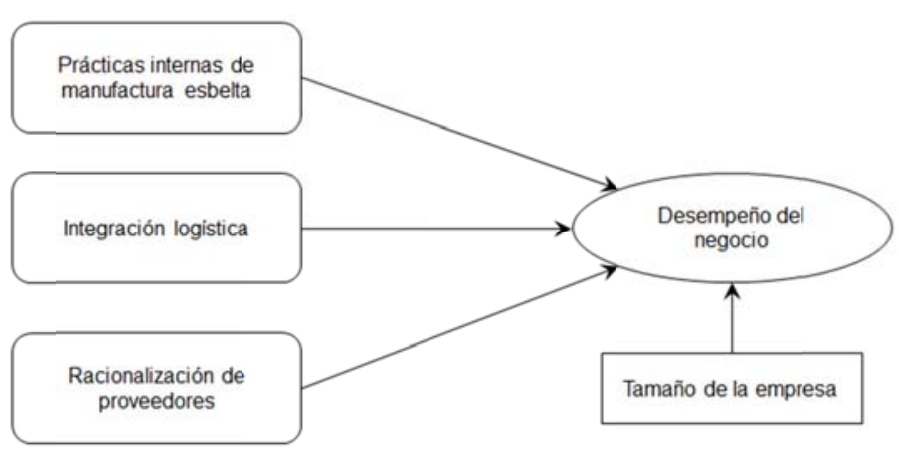

Figura 1. Marco de la investigación 


\section{MÉTODOS}

\subsection{Muestra y procedimientos}

Los datos empíricos para este estudio fueron tomados de directivos de empresas manufactureras de Australia. Los entrevistados se seleccionaron al azar de una lista comprada a una compañía de listas de correo. En total, se enviaron 1.800 encuestas y se recibieron 232 respuestas utilizables, por lo que la tasa de respuesta fue de $13.1 \%$. Dieciséis de ellas indicaron no estar interesadas, por lo que esta investigación se basó en 216 respuestas. A los encuestados se les pidió que indicaran el punto de desacoplamiento en sus sistemas de producción, en términos de MTS, MTO o ETO. Desde una perspectiva general, ETO se puede asimilar a MTO [51], por lo cual se pueden agrupar en un solo conjunto para esta muestra. De las 216 empresas, 109 tienen operaciones MTS y 107 utilizan MTO -o ETO. Los datos fueron comprobados mediante correlaciones de las respuestas y sobre la base de los sectores de la industria y el tamaño de la organización encuestada. Las pruebas $X^{2}$ en ambas categorías no indican ninguna diferencia significativa entre los dos grupos de encuestados.

En cuanto a los sectores de la industria, el $16 \%$ de los provenían de electrónica/eléctrica, el $25 \%$ de la mecanización, el $8 \%$ de la automotriz, el $11 \%$ de la industria química, el $4 \%$ de la elaboración de alimentos, el $7 \%$ de la construcción y el $12 \%$ de otros sectores industriales: equipos médicos, madera, imprenta y papel y defensa. En términos de tamaño de la organización -en función del número de empleados-, el $46 \%$ de los encuestados provenían de empresas con menos de 100 empleados, el $35 \%$ de empresas de entre 100 y 500 empleados y el $19 \%$ provenían de industrias con más de 500 empleados. Casi la mitad de los encuestados (45\%) ocupa cargos de gerentes de operaciones, el $27 \%$ de gerentes de cadena de suministro/logística, el $18 \%$ de adquisición/compra y el 3\% de gerentes de atención al cliente.

\subsection{Mediciones}

Los elementos de medición para todas las constructos se basan en los principios de investigación empírica comentados en apartados anteriores. Para las prácticas internas de manufactura esbelta se combinaron elementos de los estudios empíricos sobre producción de manufactura esbelta, incluyendo a Sakakibara et al. [29] y a Shah \& Ward [30, 31]. A los encuestados se les pidió que evaluaran el alcance de cuatro prácticas internas de manufactura esbelta aplicadas en sus empresas. Para la integración logística se adoptó la escala desarrollada por Chen \& Paulraj [52] y se focalizó en las prácticas claves necesarias para lograr una perfecta integración de las actividades logísticas con los socios de la cadena de suministro. Como respuesta a los ítems de esta escala, se les pidió a los encuestados evaluar la integración de sus actividades logísticas con sus principales asociados de la cadena de suministro, con los que las empresas tienen grandes negocios en términos de dólares. Para la racionalización de proveedores se combinaron algunos elementos de la reducción de proveedores base, propuestos por Chen \& Paulraj [52] y por Li et al. [40].

Se utilizó una escala de Likert de 7 puntos para todos los elementos de las tres escalas, con respuestas que van desde 1 -muy en desacuerdo- hasta 7 -muy de acuerdo. Para el desempeño, se les pidió a los encuestados que evaluaran el desempeño del negocio de la empresa, en comparación con el mejor competidor en el mercado, con una escala de 1 -el más débil de la industria- hasta 7 -el más fuerte en la industria. Los elementos utilizados en este estudio se detallan en la Tabla 1.

Tabla 1

Escala de validez y fiabilidad

\begin{tabular}{|c|c|c|c|}
\hline Escala & Ítem & $\begin{array}{l}\text { Factores } \\
\text { de carga }\end{array}$ & $\begin{array}{l}\text { Alfa de } \\
\text { Cronbach }\end{array}$ \\
\hline \multirow{4}{*}{$\begin{array}{l}\text { Prácticas internas } \\
\text { de manufactura } \\
\text { esbelta }\end{array}$} & $\begin{array}{l}\text { Trazo del flujo de compras para que los procesos y las máquinas estén } \\
\text { muy cerca uno del otro }\end{array}$ & 0.82 & \multirow{4}{*}{0.64} \\
\hline & $\begin{array}{l}\text { Trabajan agresivamente para reducir los tiempos de preparación en la } \\
\text { planta }\end{array}$ & 0.73 & \\
\hline & Utiliza un sistema de tracción Kanban para la producción & 0.67 & \\
\hline & Utiliza lotes pequeños de producción & 0.57 & \\
\hline \multirow{6}{*}{$\begin{array}{l}\text { Integración } \\
\text { logística externa }\end{array}$} & $\begin{array}{l}\text { Las actividades logísticas inter-organizacionales están estrechamente } \\
\text { coordinadas }\end{array}$ & 0.75 & \multirow{6}{*}{0.92} \\
\hline & $\begin{array}{l}\text { Las actividades logísticas están bien integradas con las actividades } \\
\text { logísticas de los proveedores }\end{array}$ & 0.88 & \\
\hline & $\begin{array}{l}\text { Tiene una perfecta integración de las actividades logísticas con los } \\
\text { proveedores }\end{array}$ & 0.89 & \\
\hline & $\begin{array}{l}\text { La integración logística se caracteriza por una excelente distribución, } \\
\text { transporte y/o instalaciones de almacenamiento }\end{array}$ & 0.85 & \\
\hline & $\begin{array}{l}\text { La distribución de entrada y salida de mercancías con los proveedores } \\
\text { está bien integrada }\end{array}$ & 0.78 & \\
\hline & La información y los materiales fluyen sin problemas con proveedores & 0.67 & \\
\hline \multirow{3}{*}{$\begin{array}{l}\text { Racionalización } \\
\text { de proveedores }\end{array}$} & Cuenta con un pequeño número de proveedores de alta calidad & 0.89 & \multirow{3}{*}{0.77} \\
\hline & Cuenta con un pequeño número de proveedores altamente confiables & 0.91 & \\
\hline & Mantiene proveedores que contribuyen a su desempeño competitivo & 0.68 & \\
\hline \multirow{3}{*}{$\begin{array}{l}\text { Desempeño del } \\
\text { negocio }\end{array}$} & Ventas & 0.88 & \multirow{3}{*}{0.78} \\
\hline & Retorno de la inversión & 0.78 & \\
\hline & Cuota de mercado & 0.85 & \\
\hline
\end{tabular}




\section{RESULTADOS}

\subsection{Escala de validez y fiabilidad}

Como un primer paso, las cuatro escalas fueron sometidas a un análisis de componentes principales para examinar su uni-dimensionalidad, para lo cual se siguieron los métodos empleados en [53-56]. El resultado apoya la validez de estas cuatro constructos, como lo indica su varianza, lo que supera el $50 \%$, y los factores de carga de todos los elementos dentro de cada escala superior a 0.5 [57]. El análisis de fiabilidad se llevó a cabo mediante el cálculo del coeficiente alfa de Cronbach para cada escala. El resultado muestra que las medidas de este coeficiente para los cuatro constructos supera el punto del umbral de 0.6 [58]. Los resultados del análisis factorial confirmatorio y el alfa de Cronbach se presentan en la Tabla 1.

Se utilizó una prueba de factor único de Harmann para comprobar la varianza del método común [59]. Esta prueba se realizó mediante el análisis de componentes principales y la carga de los 16 ítems en un solo factor.
La prueba verifica si del análisis de los factores puede surgir un factor único que señale la presencia de una tendencia en el método común. En el análisis factorial se obtuvo menos del $25 \%$ de la varianza y la mitad de los ítems sufrió cargas factoriales pobres, muy por debajo de 0.5. Estos resultados sugieren que la varianza de método común no era un problema importante en el conjunto de datos. Luego de reunir los requisitos de validez y de fiabilidad del constructo, se midió la calificación compuesta de cada escala mediante el cálculo de sus puntuaciones medias [57].

\subsection{Los efectos sobre el desempeño del negocio}

Los efectos de la cadena de suministro y las actividades operacionales en el desempeño empresarial se examinaron utilizando el análisis de regresión múltiple. Se incluyó el tamaño de la organización como una variable de control. El resultado se recopila en la Tabla 2.

Tabla 2

Resultados del análisis de regresión

\begin{tabular}{|c|c|c|c|}
\hline & $\begin{array}{r}\text { Varial } \\
\text { (Desem }\end{array}$ & $\begin{array}{l}\text { e dependi } \\
\text { eño del ne }\end{array}$ & $\begin{array}{l}\text { te } \\
\text { cio) }\end{array}$ \\
\hline & $\begin{array}{l}\text { Muestra total } \\
(n=216)\end{array}$ & $\begin{array}{c}\text { MTO } \\
(n=107)\end{array}$ & $\begin{array}{c}\text { MTS } \\
(n=109)\end{array}$ \\
\hline $\begin{array}{l}\text { Variable de control } \\
\text { Tamaño de la empresa }\end{array}$ & 0.07 & 0.08 & 0.06 \\
\hline $\begin{array}{l}\text { Variables independientes } \\
\text { Prácticas internas de manufactura esbelta } \\
\text { Integración de la logística externa } \\
\text { Racionalización de proveedores } \\
X^{2}\end{array}$ & $\begin{array}{l}0.20^{\star *} \\
0.19 \text { ** } \\
0.04 \\
0.13\end{array}$ & $\begin{array}{l}0.17 \\
0.39 * \star \\
-0.09 \\
0.23\end{array}$ & $\begin{array}{c}0.25^{\star \star} \\
0.03 \\
0.24^{\star} \\
0.13\end{array}$ \\
\hline
\end{tabular}

En la muestra total, tanto la integración logística como la producción de manufactura esbelta muestran una relación significativa con el desempeño del negocio. Sin embargo, la racionalización de proveedores no tiene ningún efecto sobre este desempeño cuando se considera el total de la muestra. Con base en estos hallazgos, las prácticas de manufactura esbelta y la integración logística se deben considerar como iniciativas de mejora potencial para la "edad promedio" de la planta, mientras que la racionalización de proveedores se podría descartar. Pero, ¿cuál es la diferencia entre las operaciones MTO y MTS que no aparecen cuando se considera a la totalidad de la muestra?

\subsection{Los efectos para las empresas make-to-order y make-to-stock}

La muestra se dividió en dos grupos en función del sistema de producción principal identificado por los encuestados. De las 216 empresas, 107 ejecutan sus operaciones con sistemas MTO y 109 con MTS. Estos dos grupos de empresas fueron evaluadas con respecto a las cuatro variables anteriores; los resultados se presentan en la Tabla 3 . No se observan diferencias significativas entre plantas MTO y plantas MTS con respecto a los valores medios para las prácticas de manufactura esbelta, la integración logística, la racionalización de proveedores o el desempeño del negocio. Por lo tanto, los promedios para plantas MTO y MTS no sugieren que dichas plantas hagan las cosas de manera diferente en condiciones del énfasis puesto en las diferentes iniciativas de mejora o en el nivel de desempeño del negocio que logren.

Tabla 3

Resultados de la pruebas $t$ entre plantas MTO y plantas MTS

\begin{tabular}{|l|c|c|c|}
\hline & $\begin{array}{c}\text { MTO Media } \\
\mathbf{n = 1 0 7}\end{array}$ & $\begin{array}{c}\text { MTS Media } \\
\mathbf{n = 1 0 9}\end{array}$ & $\Delta$ Media \\
\hline Prácticas de manufactura esbelta & $4.72(1.01)$ & $4.86(1.06)$ & $0.14(\mathrm{p}>0.05)$ \\
\hline Integración de la logística externa & $4.20(1.08)$ & $4.45(1.26)$ & $0.25(\mathrm{p}>0.05)$ \\
\hline Racionalización de proveedores & $5.14(1.02)$ & $5.21(0.85)$ & $0.06(\mathrm{p}>0.05)$ \\
\hline Desempeño del negocio & $4.95(1.09)$ & $4.94(1.02)$ & $0.01(\mathrm{p}>0.05)$ \\
\hline
\end{tabular}


Sin embargo, también se investigó el impacto de estas iniciativas de mejora en el desempeño del negocio utilizando el análisis de regresión múltiple para los dos sub-muestras. Los resultados se observan en la Tabla 2. Una vez más, se incluyó el tamaño de la organización como una variable de control. Las plantas MTO y MTS presentan diferencias sustanciales y significativas. Ninguna de las tres iniciativas tiene un efecto significativo para ambos tipos de plantas, pero la racionalización de proveedores tiene un efecto significativo para uno de los tipos de plantas. Sólo la integración logística muestra un efecto significativo en el desempeño del negocio para las plantas MTO; las prácticas de manufactura esbelta no muestran un efecto significativo, aunque la magnitud indica una dirección positiva. Para las plantas MTS, las prácticas de manufactura esbelta y la racionalización de proveedores muestran también efectos significativos en el desempeño del negocio, mientras que el impacto de la integración logística prácticamente es inexistente. Al comparar los resultados entre MTO y MTS, se puede inferir que los predictores de desempeño del negocio parecen estar casi en contraste.

\subsection{Comparación entre make-to-order, make-to- stock y la totalidad de la muestra}

Los resultados de la regresión para la totalidad de la muestra muestran diferencias en comparación con los dos sub-grupos muestrales -MTO y MTS-, como se observa en la Tabla 2. Las prácticas internas de manufactura esbelta tienen un impacto significativo sobre el desempeño del negocio cuando se analiza toda la muestra, lo cual apoya la hipótesis 1 . Sin embargo, en una mirada detallada a las dos submuestras es posible observar que existen diferencias significativas entre los impactos para empresas MTO y MTS. En las empresas MTS tienen un impacto significativo, mientras que el impacto para las empresas MTO no lo es tanto, lo que apoya la hipótesis 4. El coeficiente de regresión para las empresas MTS es mayor que el de toda la muestra, lo que indica que la función de las prácticas de manufactura esbelta es especialmente fuerte para las empresas MTS y mucho más fuerte que para el promedio de las empresas.

La integración logística externa es importante para el desempeño del negocio para toda la muestra, lo que apoya la hipótesis 2. Sin embargo, al analizar las dos sub-muestras se encuentran diferencias, de tal manera que el impacto sólo es significativo para las empresas MTO mientras que no se observa un impacto significativo para las empresas MTS. Este resultado apoya la hipótesis 5. La diferencia en los coeficientes de regresión es muy grande: 0.03 para MTS, 0.19 para la muestra completa y 0.39 para MTO. Esto demuestra que la integración logística tiene un impacto mucho más fuerte para las empresas MTO.

La racionalización de proveedores no tiene un impacto significativo en el desempeño del negocio cuando se estudia toda la muestra, lo cual es contrario a las expectativas teóricas. Por lo tanto, no se demuestra la hipótesis 3. Un detallado análisis MTO-MTS muestra que existe un impacto significativo para las empresas MTS, pero no para las MTO. Por lo tanto, la hipótesis 6 tampoco se demuestra. Una posible explicación radica en el diseño del producto. Las empresas MTS suelen tener diseños de productos más estables, que permiten una relación sólida con unos pocos proveedores clave durante largos períodos de tiempo, mientras que las empresas MTO pueden necesitar un conjunto más amplio de proveedores para soportar una amplia variedad de productos.

Estos resultados sugieren que los efectos de las actividades de fabricación y de la cadena de suministro en el desempeño empresarial no se generalizan a todos los tipos de empresas, debido a que los efectos para la totalidad de la muestra no se traducen en efectos correspondientes para empresas MTO y MTS. En consecuencia, esta investigación sugiere con argumentos que controlar el punto de desacoplamiento es importante para la investigación empírica en la gestión de operaciones.

\section{DISCUSIÓN DE RESULTADOS Y SUS IMPLICACIONES}

Este estudio contribuye a la investigación acerca del mejoramiento conjunto de los procesos de fabricación y de la cadena de suministro, teniendo en cuenta tanto iniciativas internas como externas orientadas en ese sentido y explorando potenciales diferencias entre empresas MTO y MTS. Específicamente, este estudio contribuye con los siguientes aspectos.

1. En general, las mejoras en los procesos, tanto internos como externos, pueden afectar el desempeño del negocio. Al analizar, en las últimas décadas lo que se ha venido desarrollando en los campos de la producción y la gestión de operaciones, se encuentra que durante los años 1980 y 1990, el énfasis se colocó en los procesos internos. Las prácticas y principios JIT y TQC se consideraban como las formas más eficaces para gestionar procesos y para producir ventaja competitiva. Más recientemente, las compañías han estado buscando por fuera de sus propias operaciones para construir nuevas competencias, es decir, gestionando su red de cadena de suministro para lograr ventajas competitivas. Al mismo tiempo, las prácticas JIT y TQC se han convertido en el concepto de manufactura esbelta. Esta investigación demuestra la contribución combinatoria de los aspectos externos e internos de las operaciones para el desempeño de la empresa.

2. Algo muy importante, los resultados muestran que existen diferencias significativas entre plantas MTO y MTS, en lo que respecta a cómo se afecta el desempeño del negocio. La integración logística externa con los proveedores es importante para plantas MTO, mientras que las prácticas internas de manufactura esbelta y la racionalización de proveedores son importantes para las plantas MTS. 
Por lo tanto, existe una distinción clara acerca de lo que crea la ventaja de negocio para este tipo de plantas.

3. Las prácticas internas de manufactura esbelta y la racionalización de proveedores, no impactan significativamente el desempeño del negocio para las plantas MTO. Las MTO manufactureras personalizan los productos a los requerimientos específicos del cliente, con flexibilidad de diseño y con entrega oportuna, lo que constituye importantes prioridades competitivas [60]. Con una amplia gama de productos y de capacidades personalizadas, el criterio para ganar por lo general no es el precio [60]. Esto implica que típicamente las plantas MTO tengan algún exceso de capacidad para efectos de la flexibilidad, reduciendo la necesidad de aplicar prácticas internas de manufactura esbelta. Las MTO manufactureras tienen múltiples proveedores de partes clave para producir variedad de productos para satisfacer las necesidades específicas de los clientes y para ofrecer una amplia gama de productos [61] y, especialmente, encargan esas partes a los proveedores después de la recepción del pedido del cliente [62]. Esto implica que la racionalización de proveedores podría llevar a una reducción en la gama de productos o del nivel de personalización que le ofrecen al cliente final.

4. El impacto de la integración logística externa sobre el desempeño empresarial no es significativo para plantas MTS. Producir productos para stock acabado implica que los volúmenes de demanda son altos y que la variabilidad de la demanda es baja - de otra manera, la planta podría no producir estos productos para el stock. El volumen por elemento y el período de tiempo no disminuyen completamente la lista de materiales -desde productos finales hasta materias primas $y$ componentes-, mientras que los volúmenes de adquisición de componentes es al menos tan alto como el de los productos finales correspondientes [63]. En consecuencia, las plantas MTS pueden utilizar relaciones arms-length con los proveedores, focalizadas en los costos y en la competencia entre proveedores, en lugar de construir integración logística con ellos.

Las implicaciones administrativas de estos resultados difieren entre plantas MTO y MTS, lo que implica que las plantas tienen que adaptar sus prácticas a la posición del punto de desacoplamiento. Las plantas MTO se enfrentan a desafíos difíciles pero importantes en el desarrollo eficaz de la integración logística con múltiples proveedores. Las MTO manufactureras producen muchos componentes desde proveedores que son esenciales para la personalización del producto, por lo cual tienen que desarrollar logística integrada con todos los proveedores que proporcionan los elementos clave. Las plantas MTS, por otro lado, puede utilizar menos proveedores de componentes estándar. Las prácticas de manufactura esbelta proporcionan una oportunidad de mejora complementaria, racionalizando las operaciones internas para una tasa de demanda estable y teniendo en cuenta una tasa de suministro igualmente estable para los proveedores.

La implicación de la investigación es que la orientación al mercado industrial, en términos de MTO versus MTS, es una variable de contingencia importante para considerar en estudios empíricos acerca de los procesos de fabricación y de las cadenas de suministro, ya que MTO y MTS están relacionadas con resultados significativamente diferentes en relación con las prácticas internas de manufactura esbelta, la integración logística externa con proveedores y la racionalización de proveedores. Los resultados de este estudio representan tres diferentes errores potenciales de interpretación, si no se reconocen las diferencias entre MTO y MTS. La práctica interna de manufactura esbelta tiene un impacto significativo en el desempeño del negocio para toda la muestra, pero no para las plantas MTO. La integración logística externa tiene un impacto significativo en el desempeño del negocio para toda la muestra, pero no para las plantas MTS. Por último, la racionalización de proveedores no tiene un impacto significativo en el desempeño del negocio para toda la muestra, pero sí para plantas MTS. Estas reflexiones muestran que la distinción entre MTO y MTS puede ser un factor de contingencia importante a tener en cuenta cuando se analizan los resultados de la encuesta en los que se incluyen la muestra total y las sub-muestras MTO y MTS.

\section{LIMITACIONES Y TRABAJO FUTURO}

Una limitación de este estudio es la población de la muestra, que se limita a las empresas australianas. Aunque se espera que estos resultados mantengan las cadenas de fabricación y de suministro en general, no se puede afirmar que este sea el caso. Por lo tanto, la investigación futura podría extender este estudio a una población más amplia de empresas, incluyendo a otros países, para la generalización de los resultados y para detectar posibles efectos-país. Las diferencias potenciales entre plantas MTO y MTS probablemente no se restringen a las tres áreas de este estudio, por lo que se requieren más estudios de investigación acerca de otros factores para detectar otras diferencias. Se notó la falta de investigación acerca de la racionalización de proveedores con respecto a la diferenciación potencial entre empresas MTO y MTS, para lo cual los estudios de caso y la investigación por encuestas podrían contribuir con la base de conocimiento.

\section{CONCLUSIONES}

Este estudio presenta resultados de una investigación mediante encuesta acerca de las diferencias entre plantas MTO y MTS. Mientras que las iniciativas de mejora para las cadenas de fabricación y de suministro tienen un impacto significativo en el desempeño del negocio, existen diferencias igualmente significativas entre las plantas MTO y MTS. Las plantas MTO se benefician de la integración logística con los 
proveedores externos, mientras que las plantas MTS se benefician de las prácticas internas de manufactura esbelta y de la racionalización de proveedores. Por lo tanto, la diferenciación entre plantas MTO y MTS ofrece un factor de contingencia importante en la investigación acerca de ambas cadenas.

\section{REFERENCIAS}

[1] J. P. Womack \& D. T. Jones. "Lean Thinking". Simon \& Schuster, 1996.

[2] J. B. Naylor; M. M. Naim \& D. Berry. "Leagility: integrating the lean and agile manufacturing paradigms in the total supply chain". International Journal of Production Economics, Vol. 62, No. 1-2, pp.107-18, 1999.

[3] T. van der Vaart \& D. P. van Donk. "A critical review of survey-based research in supply chain integration". International Journal of Production Economics, Vol. 111, No. 1, pp. 42-55, 2008.

[4] F. Sahin \& E. P. Robinson. "Information sharing and coordination in make-to- order supply chains". Journal of Operations Management, Vol. 23, No. 6, pp. 579-598, 2005.

[5] G. Quesada et al. "Linking order winning and external supply chain integration strategies". Supply Chain Management: An International Journal, Vol. 13, No. 4, pp. 296-303, 2008.

[6] J. J. Chen \& A. Paulraj. "Understanding supply chain management: critical research and a theoretical framework". International Journal of Production Research, Vol. 42, No. 1, pp. 131-163, 2004.

[7] P. R. Lawrence \& J. W. Lorsch. "Organization and environment: managing differentiation and integration". Harvard Business School Press, 1986.

[8] J. D. Thompson. "Organizations in action: Social Science Bases of Administrative Theory". McGraw-Hill, 1967.

[9] R. Sousa \& C. A Voss. "Contingency research in operations management practices". Journal of Operations Management, Vol. 26, No. 6, pp. 697-713, 2009.

[10] B. B. Flynn; B. Huo \& X. Zhao. "The impact of supply chain integration on performance: A contingency and configuration approach". Journal of Operations Management, Vol. 28, No. 1, pp. 58-71, 2010.

[11] P. Joynt. "Contingency analysis for effective administration". The International Journal of Management Science, Vol. 5, No. 4, pp. 425-435, 1977.

[12] T. Burns \& G. M. Stalker. "The Management of Innovation". Social Science Paperbacks, 1961.

[13] L. Hendry. "Applying world class manufacturing to maketo-order companies: problems and solutions". International Journal of Operations and Production Management, Vol. 18, No. 11, pp. 1086-1100, 1998.

[14] W. L. Berry \& T. Hill. "Linking systems to strategy". International Journal of Operations and Production Management, Vol. 12, No. 1, pp. 3-15, 1992.

[15] A. J. D'Alessandro \& A. Baveja. "Divide and conquer: Rohm and Haas' response to a hanging specialty chemicals market". Interfaces, Vol 30 , No. 6, pp. 1-16, 2000.

[16] D. P. Van Donk. "Make to stock or make to order: the decoupling point in the food processing industries". International Journal of Production Economics, Vol. 69, No. 2, pp. 297-306, 2001.

[17] A. Arreola-Risa \& G. A. DeCroix. "Make-to-order versus make-to-stock in a production-inventory system with general production times". IIE Transactions, Vol. 30, pp. 705-713, 1998.
[18] A. Federgruen \& Z. Katalan."The impact of adding a make-to-order item to a make-to-stock system". Management Science, Vol. 45, No. 7, pp. 980-994, 1999.

[19] S. Rajagopalan. "Make to order or make to stock: model and application". Management Science, Vol. 48, No. 2, pp. 241-256, 2002.

[20] D. Gupta \& S. Benjaafar. "Make-to-order, make-to-stock, or delayed product differentiation? A common framework for modeling and analysis". IIE Transactions, Vol. 36, pp. 529-546, 2004.

[21] J. A. Gonzalez-Benito. "A theory of purchasing's contribution to business performance". Journal of Operations Management, Vol. 25, No. 4, pp. 901-917, 2007.

[22] V. R. Kannan \& K. C. Tan. "Supplier selection and assessment: Their impact on business performance". Journal of Supply Chain Management, Vol. 35, No. 4, pp. 11-21, 2002.

[23] V. R. Kannan \& K. C. Tan. "Just in time, total quality management, and supply chain management: understanding their linkages and impact on business performance". The International Journal of Management Science, Vol. 33, No. 2, pp. 153-162, 2005.

[24] E. D. Rosenzweig; A. V. Roth \& J. W. Dean. "The influence of an integration strategy on competitive capabilities and business performance: an exploratory study of consumer products manufacturers". Journal of Operations Management, Vol. 21, No. 4, pp. 437-456, 2003.

[25] F. M. Tseng; Y. J. Chiu \& J. S. Chen. "Measuring business performance in the high- tech manufacturing industry: A case study of Taiwan's large-sized TFT-LCD panel companies". The International Journal of Management Science, Vol. 37, pp. 686-697, 2009.

[26] J. F. Krafcik. "Triumph of the lean production systems". Sloan Management Review, Vol. 30, No. 1, pp. 41-52, 1988.

[27] J. P. Womack; D. T. Jones \& D. Roos. "The Machine that Changed the World: The Story of Lean Production". New York: Simon \& Schuster, 1990.

[28] R. J. Schonberger. "Japanese production management: an evolution-with mixed success". Journal of Operations Management, Vol. 25, No. 2, pp. 403-419, 2007.

[29] S. Sakakibara; B. B. Flynn \& R. G. Schroeder. "A framework and measurement instrument for just-in-time manufacturing". Production and Operations Management, Vol. 2, No. 3, pp. 177-194, 1993.

[30] R. Shah \& P. T. Ward. "Lean manufacturing: context, practice bundles, and performance". Journal of Operations Management, Vol. 21, No. 2, pp. 129-149, 2003.

[31] R. Shah \& P. T. Ward. "Defining and developing measures of lean production". Journal of Operations Management, Vol. 25, No. 4, pp. 785-805, 2007.

[32] J. L. Callen; C. Fader \& I. Krinsky. "Just in time: a cross sectional plant analysis". International Journal of Production Economics, Vol. 63, No. 3, pp. 277-301, 2000.

[33] R. R. Fullerton \& C. S. McWatters. "The production performance benefits from JIT implementation". Journal of Operations Management, Vol. 19, No. 1, pp. 81-96, 2001.

[34] M. Huson \& D. Nanda. "The impact of just in time manufacturing on firm performance in the US". Journal of Operations Management, Vol. 12, No. 3-4, pp. 297-310, 1995.

[35] R. Germain. "The role of context and structure in radical and incremental logistics innovation adoption". Journal of Business Research, Vol. 35, No. 2, pp. 117-127, 1996. 
[36] R. Germain \& C. Droge. "The context, organizational design, and performance of JIT versus non JIT buying firms". International Journal of Purchasing and Materials Management, Vol. 34, No. 2, pp. 12-18, 1998.

[37] M. T. Frohlich \& R. Westbrook. "Arcs of integration: an international study of supply chain strategies". Journal of Operations Management, Vol. 19, No. 2, pp. 185-200, 2001.

[38] A. Seidmann \& A. Sundararajan. "The effects of task and information asymmetry on business process redesign". International Journal of Production Economics, Vol. 50, No. 2-3, pp. 117-128, 1997.

[39] A. De Toni \& G. Nassimbeni. "Buyer-supplier operational practices, sourcing policies and plant performances: results of an empirical research". International Journal of Production Research, Vol. 37, No. 3, pp. 597-619, 1999.

[40] S. Li et al. "Development and validation of a measurement instrument for studying supply chain management practices". Journal of Operations Management, Vol. 23, No. 6, pp. 618-641, 2005.

[41] P. D. Berger: A. Gerstenfeld \& A. Z. Zeng. "How many suppliers are best? A decision-analysis approach". The International Journal of Management Science, Vol. 32, No. 1, pp. 9-15, 2004.

[42] H. Yu; A. Z. Zeng \& L. Zhao. "Single or dual sourcing: decision-making in the presence of supply chain disruption risks". The International Journal of Management Science, Vol. 37, No. 4, pp. 788-800, 2009.

[43] R. Narasimhan; S. Talluri \& D. Mendez. "Supplier evaluation and rationalization via data envelopment analysis: an empirical examination". Journal of Supply Chain Management, Vol. 37, No. 3, pp. 28-37, 2001.

[44] R. Mason-Jones; B. Naylor \& D. R. Towill. "Lean agile or leagile? Matching your supply chain to the marketplace". International Journal of Production Research, Vol. 38, No. 17, pp. 4061-4070, 2000.

[45] R. Krishnamurthy \& C. A. Yauch. "Leagile manufacturing: a proposed corporate infrastructure". International Journal of Operations and Productions Management, Vol. 27, No. 6, pp. 588-604, 2007.

[46] M. Hallgren \& J. Olhager. "Lean and agile manufacturing: external and internal drivers and performance outcomes". International Journal of Operations and Production Management, Vol. 29, No. 10, pp. 976-999, 2009.

[47] J. Riezebos; W. Klingenberg \& C. Hicks. "Lean production and information technology: connection or contradiction?" Computers in Industry, Vol. 60, No. 4, pp. 237-247, 2009.

[48] R. E. White \& V. Prybutok. "The relationship between JIT practices and type of production system". Omega, Vol. 29, No. 2, pp. 113-124, 2001.

[49] M. A. Cusumano. "The limits of lean". Sloan Management Review, Vol. 35, No. 4, pp. 27-32, 1994.
[50] S. Zailani \& P. Rajagopal. "Supply chain integration and performance: US versus East Asian companies". Supply Chain Management: An International Journal, Vol. 10, No. 5, pp. 379-393, 2005.

[51] J. Olhager. "Strategic positioning of the order penetration point". International Journal of Production Economics, Vol. 85, No. 3, pp. 319-329, 2003.

[52] I. J. Chen \& A. Paulraj. "Towards a theory of supply chain management: the constructs and measurements". Journal of Operations Management, Vol. 22, No. 2, pp. 119-150, 2004.

[53] B. B. Flynn; R. G. Schroeder \& S. Sakakibara. "A framework for quality management research and an associated measurement instrument". Journal of Operations Management, Vol. 11, No. 4, pp. 339-366, 1994.

[54] P. T. Ward et al. "Business environment, operations strategy, and performance: An empirical study of Singapore manufacturers". Journal of Operations Management, Vol. 13, No. 2, pp. 99-115, 1995.

[55] S. M. Meyer \& D. A. Collier. "An empirical test of the causal relationships in the Baldrige Health Care Pilot Criteria". Journal of Operations Management, Vol. 19, No. 4, pp. 403-426, 2001.

[56] X. A. Koufteros; M. A. Vonderembse \& W. J. Doll. "Integrated product development practices and competitive capabilities: the effects of uncertainty, equivocality, and platform strategy". Journal of Operations Management, Vol. 20, No. 4, pp. 331-355, 2002.

[57] J. F. Hair et al. "Multivariate Data Analysis". Prentice-Hall International Inc., 1998.

[58] J. Nunnally. "Psychometric Theory". McGraw-Hill, 1978.

[59] P. M. Podsakoff \& D. Organ. "Self-reports in organizational research". Journal of Management, Vol. 12, No. 4, pp. 531-544, 1986.

[60] A. Hill \& T. Hill. "Manufacturing Operations Strategy". Palgrave Macmillan Houndsmills, 2009.

[61] T. Sawik. "Single vs. multiple objective supplier selection in a make to order environment". The International Journal of Management Science, Vol. 38, No. 3-4, pp. 203-212, 2010.

[62] J. Yue; Y. Xia \& T. Tran. "Selecting sourcing partners for a make-to-order supply chain". The International Journal of Management Science, Vol. 38, No. 3-4, pp. 136-144, 2010.

[63] J. Olhager. "Supply chain management: a just-in-time perspective". Production Planning and Control, Vol. 13, No. 8, pp. 681-687, 2002. 